\title{
Ultra-High Efficient Thermal Tuning of Dielectric Optical Waveguides
}

\author{
Faisal Ahmed Memon ${ }^{1,2}$, Francesco Morichetti ${ }^{1}$ and Andrea Melloni ${ }^{1}$ \\ ${ }^{I}$ Dipartimento di Elettronica, Informazione e Bioingegneria (DEIB), Politecnico di Milano, via Ponzio 34/5, 20133 Milan Italy \\ ${ }^{2}$ Department of Telecommunications Engineering, Mehran University of Engineering \& Technology Jamshoro 76062 Pakistan \\ faisalahmed.memon@polimi.it
}

\begin{abstract}
We show that high-refractive index silicon oxycarbide (SiOC) exhibits a record thermooptic coefficient $\left(2.5 \times 10^{-4}{ }^{\circ} \mathrm{C}^{-1}\right)$, about $30 \times$ larger than that of silica, enabling the realization of low-power-consumption thermally-tunable dielectric photonic platforms.

OCIS codes: (230.3120) Integrated optics devices; (160.4670) Optical materials
\end{abstract}

\section{Introduction}

With respect to semiconductor photonic platforms, such as $\mathrm{Si}$ and InP, dielectric platforms, like germanium-doped silica $\left(\mathrm{Ge}: \mathrm{SiO}_{2}\right)$, silicon nitride $(\mathrm{SiN})$ and silicon oxynitride $(\mathrm{SiON})$, offer lower loss, lower polarization dependence, and more efficient fiber-coupling. Active tuning of photonics integrated circuits (PICs) fabricated with these materials is typically achieved through thermo-optic effect, which however has a very low efficiency because of the low thermo-optic coefficient (TOC), that is on the order of $10^{-5}{ }^{\circ} \mathrm{C}^{-1}$. As a result, thermally actuated PICs in $\mathrm{SiO}_{2}, \mathrm{SiON}$ and $\mathrm{Si}_{3} \mathrm{~N}_{4}$ show a limited frequency shift versus temperature $\left(\sim 1 \mathrm{GHz} /{ }^{\circ} \mathrm{C}\right)$ and require long $(\mathrm{mm}$-scale $)$ thermal actuators, high electrical power (some hundreds of $\mathrm{mW}$ for $\pi$ phase shift) [1], and high operational temperatures, which also imply severe thermal crosstalk issues.

Silicon oxycarbide ( $\mathrm{SiOC}$ ) is a glass compound that has recently found applications as interlayer dielectric, anode material in ion batteries and photoluminescence [2-4]. Recently, we have demonstrated that SiOC is a suitable material for integrated photonics, because of the wide tuneability of the refractive index $n$ and low absorption in near-infrared range, enabling the realization of high-index-contrast low-loss waveguides [5].

In this work, we show that high-refractive index SIOC $(n=2.2)$ exhibits a record TOC as high as $2.5 \times \cdot 10^{-4}{ }^{\circ} \mathrm{C}^{-1}$, that is about 30 times larger than that of silica and almost twice that of $\mathrm{Si}$. Not only this makes SiOC a promising platform for low-power consumption dielectric PICs, but we also demonstrate the integration of SiOC films in classical dielectric waveguides to enhance the efficiency of thermo-optic tuning.

\section{SiOC technology and waveguide fabrication}

The SiOC films employed in this work were deposited by reactive RF magnetron sputtering from a $\mathrm{SiC}$ target in $\mathrm{O}_{2}$ and $\mathrm{Ar}$ atmosphere, according to the process described in [5]. As shown in Fig. 1(a), by changing the process parameters (RF power and $\mathrm{O}_{2}$ flow), the $\mathrm{SiOC}$ refractive index can be tuned from that of $\mathrm{SiO}_{2}(1.45)$ to that of $\mathrm{SiC}$ (above 3) by increasing the $\mathrm{C}$ content. To maintain transparency in the neat-IR range [see Fig. 1(b)], we selected a material composition of $\mathrm{Si}_{0.45} \mathrm{O}_{0.27} \mathrm{C}_{0.27}$, as measured by X-ray photoelectron spectroscopy (XPS), resulting in a refractive index of 2.2 in the near-IR range. Optical waveguides were fabricated by depositing a 175-nm-thick SiOC film (core) on a silica substrate. The waveguide cross section was defined by direct-laser-writing lithography and reactive ion etching in $\mathrm{CHF}_{3}(100 \mathrm{sccm})$ and $\mathrm{O}_{2}(5 \mathrm{sccm})$. Figure 1 (c) shows the waveguide cross section before the
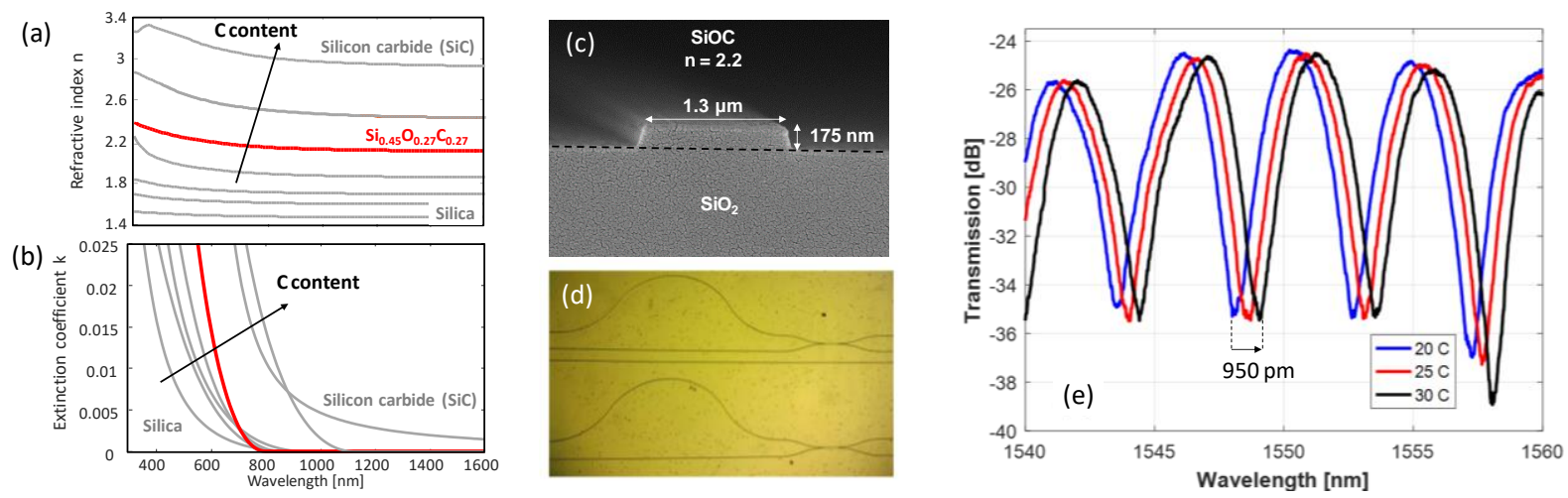

Fig. 1. Measured (a) refractive index and (b) extinction coefficient of SiOC films with increasing carbon composition. (c) SEM image of a SIOC strip waveguide before $\mathrm{SiO}_{2}$ upper cladding deposition. (d) Top view photograph of MZIs fabricated with SiOC strip waveguides. (e) Thermally induced wavelength shift of the transmission of a SiOC MZI. 
(a)

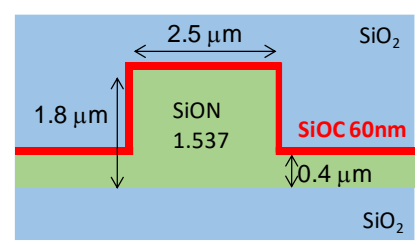

(b)

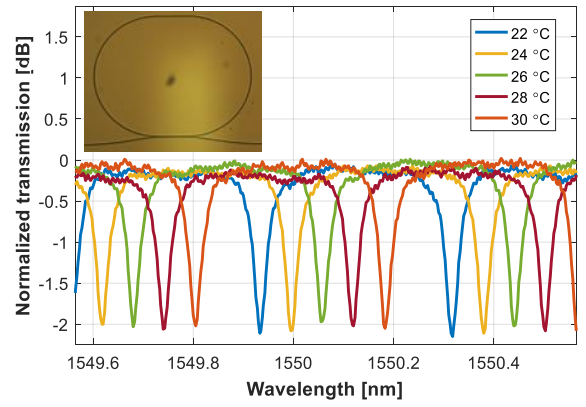

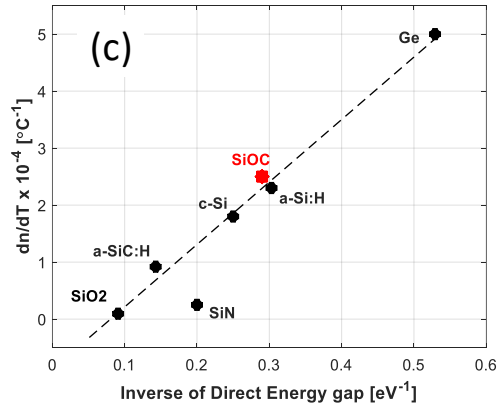

Fig. 2. (a) Schematic cross-section of a SiOC-coated $\mathrm{SiON}$ rib waveguide. (b) Thermally induced wavelength shift of the transmission of a $\mathrm{SiOC}-$ coated SiON racetrack resonator (device picture in the inset). (c) Dependence of the TOC of several materials on the inverse of the direct-band-gap energy.

upper cladding deposition. After the SiOC core patterning, PECVD silica $(n=1.45)$ was deposited as upper cladding material. At a wavelength $\lambda_{0}=1550 \mathrm{~nm}$, the strip shaped waveguide has a group index $n_{\mathrm{g}}=1.93$ and propagation losses of $2 \mathrm{~dB} / \mathrm{cm}$ for transverse electric (TE) polarized light.

\section{Thermo-optic coefficient of SiOC-based waveguides}

The TOC of the fabricated SIOC strip waveguides was evaluated by measuring the thermally-induced wavelength shift of unbalanced Mach-Zehnder interferometers (MZIs). To this aim, we used the devices shown in Fig. 1(b), which were designed with a waveguide unbalance of about $240 \mu \mathrm{m}$ in order to exhibit a free spectral range (FSR) of $5 \mathrm{~nm}$. Figure 1(e) shows the measured transmission of one of the fabricated MZIs around $1550 \mathrm{~nm}$, when the temperature $T$ is increased from $25{ }^{\circ} \mathrm{C}$ to $35{ }^{\circ} \mathrm{C}$ by using a thermoelectric cooler underneath the sample. The observed wavelength shift $\mathrm{d} \lambda / d T$ is as large as $95 \mathrm{pm} /{ }^{\circ} \mathrm{C}$, which corresponds to an effective TOC of the waveguide $K_{\text {eff }}=n_{\mathrm{g}} \mathrm{d} \lambda / \lambda_{0} d T=1.2 \times 10^{-4}{ }^{\circ} \mathrm{C}^{-1}$ that is one order of magnitude larger than typical dielectric platforms. The TOC of the $\mathrm{SiOC}$ material is evaluated by considering the confinement factor of the optical mode in all the materials (SiOC, $\mathrm{SiON}, \mathrm{SiO}_{2}$ ) the waveguide is made of. As a result, we found that the TOC of $\mathrm{SiOC}, K_{S i O C}=2.5 \times 10^{-4}{ }^{\circ} \mathrm{C}^{-1}$, is the largest ever reported for a dielectric material employed in optical waveguides.

The high TOC of SiOC can be exploited to enhance the temperature sensitivity of PICs fabricated with conventional dielectric platforms. Here, we report on the integration of a SiOC film on a SiON waveguide, according to the cross-sectional scheme shown in Fig. 2(a). A 60-nm-thin SiOC layer $(n=2.2)$ was deposited on a $\mathrm{SiON}$ core rib-shaped waveguide $\left(2.5 \mu \mathrm{m} \times 1.8 \mu \mathrm{m}, 0.4 \mu \mathrm{m}\right.$ side slab), which was then buried under a $\mathrm{SiO}_{2}$ cladding. All-pass racetrack resonators [see inset of Fig. 2(b)] were fabricated by using SIOC-coated SiON waveguides. Transmission measurements in Fig. 2(b) show a resonance shift by $250 \mathrm{pm}$ when the temperature increases from $22{ }^{\circ} \mathrm{C}$ to $30{ }^{\circ} \mathrm{C}$, resulting in a $K_{\text {eff }}$ is $3.2 \times 10^{-5}{ }^{\circ} \mathrm{C}^{-1}$. Remarkably, a thin layer of SiOC of only $60 \mathrm{~nm}$ increases by three times the temperature sensitivity of the native $\mathrm{SiON}$ waveguide $\left(K_{S i O N}=1.1 \times 10^{-5}{ }^{\circ} \mathrm{C}^{-1}\right)$.

The origin of the large TOC of SiOC is related to the increase of the dielectric polarizability near the transparency edge [6]. Figure 2(c) shows the TOC of various materials (black marks, data from literature [7]), which is nearly dependent on the inverse of $E_{\mathrm{g}}$ and increases approaching the band gap. Ge has the highest TOC but it is not transparent in the near-IR, while the high transparency of $\mathrm{SiO}_{2}$ till the deep UV is associated with a very small TOC. The high TOC of SiOC (red mark), whose direct bandgap is around $E_{\mathrm{g}}=3.5 \mathrm{eV}$, as derived from absorption spectra of Fig. 1(b), is well in line with this trend. This also shows that the SiOC composition employed in this work offer a good tradeoff between material transparency and high TOC.

In conclusion, our results show that high-refractive-index $\mathrm{SiOC}$ is a promising material for the realization of low-power-consumption thermally-tunable dielectric photonic platforms.

This work was partially funded by Fondazione Cariplo Project “Advanced Control Technologies for Integrated Optics (ACTIO)” Rif. $2016-0881$.

\section{References}

[1] P. Muñoz et al, "Silicon nitride photonic integration platforms for visible, near-infrared and mid-infrared applications," Sensors, 17, 2088

(2017).

[2] P. Mandracci et al, "Optical and structural properties of amorphous silicon-nitrides and silicon-oxycarbides: Application of multilayer structures for the coupling of Bloch Surface Waves," Journal of Non-Crystalline Solids, 453, 113-117, (2016).

[3] L. David et al, "Silicon oxycarbide glass-graphene composite paper electrode for long-cycle lithium-ion batteries," Nat. Comm., 7, 10998

(2016)

[4] V. Nikas et al, "The origin of white luminescence from silicon oxycarbide thin films," App. Phys. Letters, 104, 061906 (2014)

[5] F. A. Memon et al, "Waveguiding light into silicon oxycarbide," Appl. Sci., 7(6), 561 (2017)

[6] M. Bass, Handbook of Optics: Volume IV - Optical Properties of Materials, Nonlinear Optics, Quantum Optics, (McGraw-Hill 2010).

[7] E. D. Palik, Handbook of thermo-optic coefficients of optical materials with applications (Academic, 1998). 